\title{
On Kalman Active Observers
}

\author{
Rui Cortesão
}

Received: 12 November 2004 / Accepted: 2 May 2006 /

Published online: 27 October 2006

(C) Springer Science + Business Media B.V. 2006

\begin{abstract}
The paper introduces the Active Observer (AOB) algorithm in the framework of Kalman filters. The AOB reformulates the Kalman filter to accomplish model-reference adaptive control based on: (1) A desired closed loop system. (2) An extra equation to estimate an equivalent disturbance referred to the system input. An active state is introduced to compensate unmodeled terms, providing a feedforward compensation action. (3) Stochastic design of the Kalman matrices. Stability analysis with model errors is discussed. An example of robot force control with an external and unknown nonlinear disturbance is presented (SISO system). Another example of model-matching control for steer-by-wire (SBW) vehicles with underactuated structure is discussed (MIMO system).
\end{abstract}

Key words disturbances · Kalman filter · observers · state space control · stochastic estimation

\section{Introduction}

In complex control systems, unknown disturbances, higher order dynamics, nonlinearities and noise are always present. All these disturbances may arise in a dynamical model due to the intrinsic nature of the system or deliberately introduced by external sources. A great variety of methods and techniques have been proposed to deal with disturbances. De Schutter [20] has proposed an extended deterministic observer to estimate the motion parameters of a moving object in a force control task. In [5], model uncertainties, nonlinearities and external disturbances are merged to one term and then compensated with a nonlinear disturbance observer based on the variable structure system theory. Several drawbacks of previous methods are also pointed

\footnotetext{
R. Cortesão $(\bowtie)$

Institute of Systems and Robotics, Electrical and Computer Engineering Department, University of Coimbra - Campus 2, 3030-290 Coimbra, Portugal

e-mail: cortesao@isr.uc.pt
} 
out in [5]. The problem of disturbance decoupling is classical and occupies a central role in modern control theory. Many control problems including robust control, decentralized control and model reference control can be recast as an almost disturbance decoupling problem. To tackle the disturbance decoupling problem, PIDbased techniques [15], state feedback [6] geometric concepts [8], tracking schemes [4] and observer techniques [17] have been proposed among others. In the AOB, the disturbance estimation is modeled as an auto-regressive (AR) process with fixed parameters driven by a random source. This process represents stochastic evolutions. The AOB provides a methodology to achieve model-reference adaptive control through extra states and stochastic design. It has been applied in several robotic applications, such as autonomous compliant motion of robotic manipulators $[10,11$, 18], haptic manipulation [13], humanoids [19], and mobile systems [2, 7, 9, 16].

The paper is organized as follows: This section briefly discusses previous work, pointing out the differences of the AOB approach. The AOB concept including the AOB structure is discussed in Section 2. The AOB design is analyzed in Sections 3 and 4 for the first-order AOB (AOB-1) and $N^{t h}$-order AOB (AOB-N), respectively. Estimation strategies are discussed in Section 5. Stability analysis is made in Section 6. Before the conclusions, two example are presented. The first one discusses robot force control (SISO system). The second one addresses model-matching control for SBW vehicles with underactuated structure (MIMO system).

\section{AOB Concept}

A linear system represented in state space can be controlled through state feedback (e.g. optimal control, adaptive control and explicit pole placement control). In practice, the main problem of this approach is that unmodeled terms including noise, higher order dynamics, parameter mismatches and unknown disturbances are not addressed in the control design. Therefore, it is necessary to develop a control structure that can deal with them, to boost control performance. The main goal of the AOB is to achieve model-reference adaptive control using Kalman techniques. To accomplish this goal, a description of the system in closed loop and open loop is necessary. The motivation for the AOB, discussed in the sequel, is based on:

1. A desired closed loop system.

2. An extra equation to estimate an equivalent disturbance referred to the system input. An active state active state $p_{k}$ (extra-state) is introduced to compensate

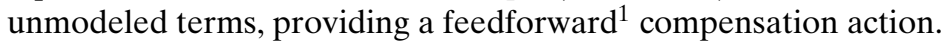

3. The stochastic design of the Kalman matrices $Q_{k}$ (model uncertainty) and $R_{k}$ (measure uncertainty) for the AOB context.

The AOB can be applied to systems modeled by linear equations plus an equivalent disturbance referred to the input. Modeling nonlinear systems by nominal nonlinear systems plus disturbances has not been considered yet, but it is one of the goals for the future.

1 The word "feedforward" is used to emphasize the fact that the AOB is generating an "external" input to act on the system.

悬 Springer 


\subsection{AOB Structure}

Given a system with equations

$$
x_{r, k}=\Phi_{r} x_{r, k-1}+\Gamma_{r} u_{k-1}+\xi_{x_{r, k}}
$$

and

$$
y_{k}=C_{r} x_{r, k}+\eta_{k}
$$

an observer of the state $x_{r, k}, \hat{x}_{r, k}$, can be written as

$$
\hat{x}_{r, k}=\Phi_{r, n} \hat{x}_{r, k-1}+\Gamma_{r, n} u_{k-1}+K_{k}\left[y_{k}-C_{r}\left(\Phi_{r, n} \hat{x}_{r, k-1}+\Gamma_{r, n} u_{k-1}\right)\right],
$$

where $\Phi_{r, n}$ and $\Gamma_{r, n}$ are, respectively, the nominal state transition and command matrices (i.e., the ones used in the design). $\Phi_{r}$ and $\Gamma_{r}$ are the real matrices. $\xi_{x_{r, k}}$ and $\eta_{k}$ are Gaussian random variables associated to the system and measures, respectively, having a key role in the AOB design. Defining the estimation error as

$$
e_{r, k}=x_{r, k}-\hat{x}_{r, k},
$$

and considering ideal conditions (i.e., the nominal matrices are equal to the real ones and $\xi_{x_{r, k}}$ and $\eta_{k}$ are zero), $e_{r, k}$ can be computed from Eqs. 1 and 3. Its value is

$$
e_{r, k}=\left(\Phi_{r}-K_{k} C_{r} \Phi_{r}\right) e_{r, k-1} .
$$

The error dynamics given by the eigenvalues of $\left(\Phi_{r}-K_{k} C_{r} \Phi_{r}\right)$ is function of the $K_{k}$ gain. The Kalman observer computes the best $K_{k}$ in a straightforward way, minimizing the mean square error of the state estimate due to the random sources $\xi_{x_{r, k}}$ and $\eta_{k}$. When there are unmodeled terms, Eq. 5 needs to be changed. A deterministic description of $e_{r, k}$ is difficult, particularly when unknown modeling errors exist. Hence, a stochastic approach is attempted to describe it. If state feedback from the observer is used to control the system, an additional input $p_{k}$ enters in the system,

$$
p_{k}=-L_{r} e_{r, k},
$$

where $L_{r}$ is the state feedback gain. A state space equation should be found to characterize this undesired input, leading the system to an extended state representation.

The classical observer depicted in Figure 1 cannot detect $p_{k}$. The observer receives $u_{k}$ given by

$$
u_{k}=r_{k}-L_{r} x_{r, k}+p_{k} .
$$

From $u_{k}$, it is not possible to distinguish $p_{k}$ from $r_{k}$. To overcome this difficulty, the observer ought to know $r_{k}$. Figure 2 shows the AOB. The active state $\hat{p}_{k}$ estimates the extended state $p_{k}$ permitting the overall system to have the desired behavior.

2 The name "active state" is used for both $p_{k}$ and $\hat{p}_{k} \cdot p_{k}$ can be seen as an equivalent disturbance state that exists in the real system, and $\hat{p}_{k}$ is the estimation of $p_{k}$. 


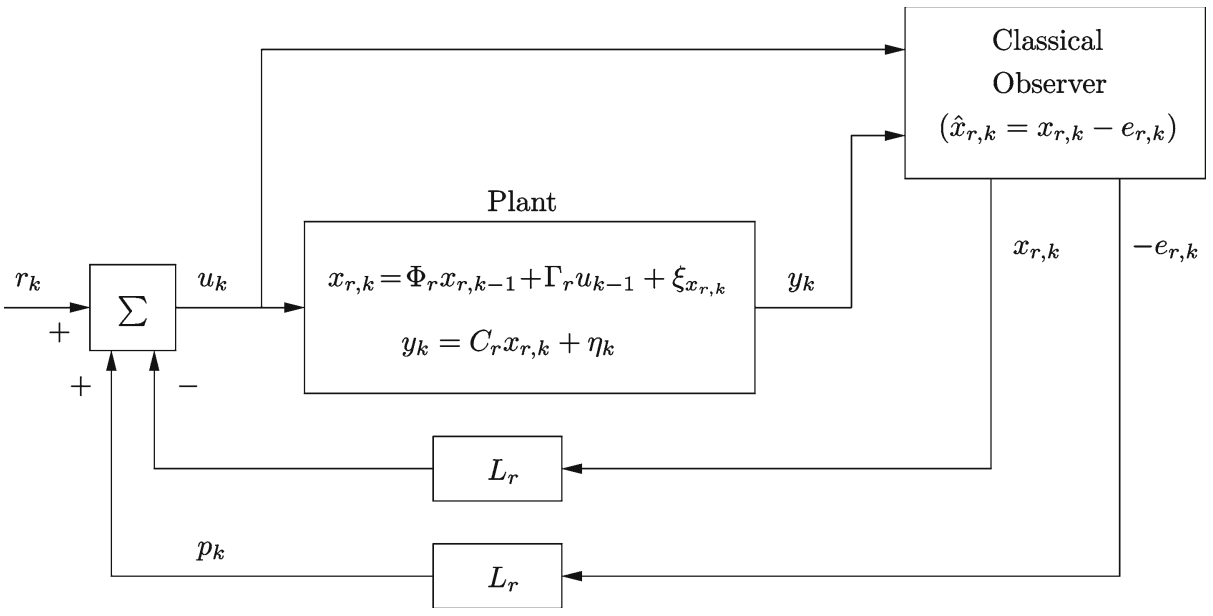

Figure 1 Classical observer. The observer error $-e_{r, k}$ enters in the system as an additional input through the state feedback gain $L_{r}$.

\subsection{Active State Equations}

The $p_{k}$ equation has a key role in the AOB structure, crossing the AOB design. To be able to track functions with unknown dynamics, a stochastic equation is used to describe $p_{k}$ :

$$
p_{k}-p_{k-1}=\xi_{p_{k}},
$$

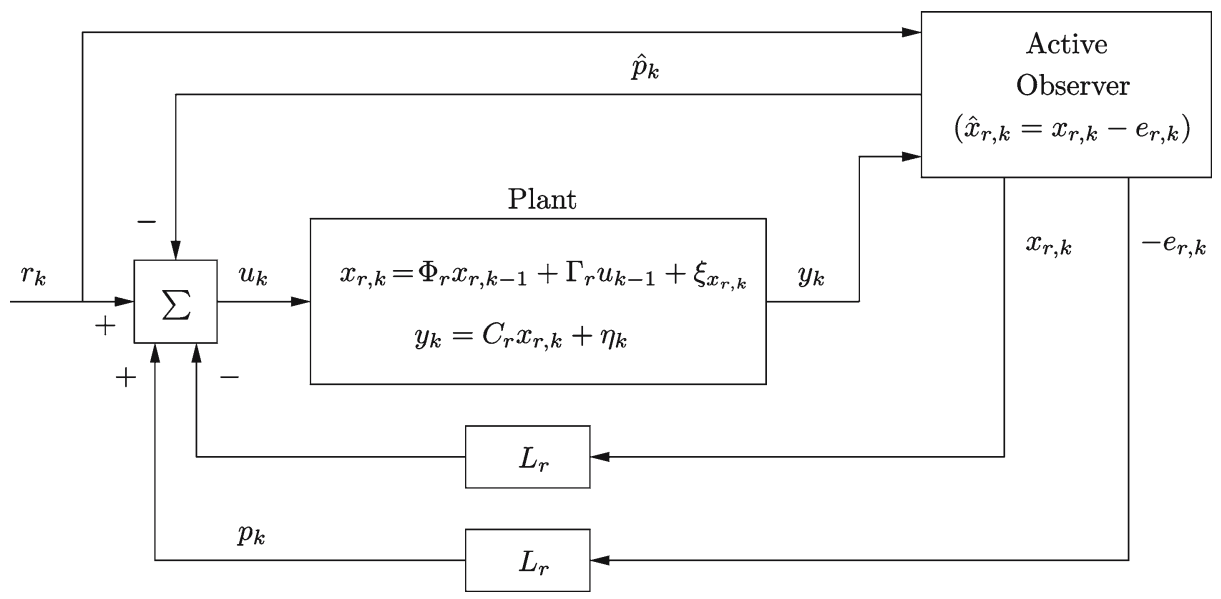

Figure 2 Active observer. The active state $\hat{p}_{k}$ compensates the error $e_{r, k}$ referred to the system input, which is described by $p_{k}$. 
in which $\xi_{p_{k}}$ is a zero-mean Gaussian random variable. ${ }^{3}$ Equation 8 says that the first derivative (or first-order evolution) of $p_{k}$ is randomly distributed. Defining ${ }^{N} \xi_{p_{k}}$ as the $N^{t h}$-order evolution of $\xi_{p_{k}}$ (or the $(N+1)^{t h}$-order evolution of $p_{k}$ ),

$$
{ }^{N} \xi_{p_{k}}={ }^{N-1} \xi_{p_{k}}-{ }^{N-1} \xi_{p_{k-1}} \text {, with }{ }^{0} \xi_{p_{k}}=\xi_{p_{k}},
$$

the general form of Eq. 8 is

$$
p_{k}=\sum_{j=1}^{N}(-1)^{j+1} \frac{N !}{j !(N-j) !} p_{k-j}+{ }^{N-1} \xi_{p_{k}} .
$$

$\left\{p_{n}\right\}$ is an AR process ${ }^{4}$ of order $N$ with undetermined mean. It has fixed parameters given by Eq. 10 and is driven by the statistics of $\left\{{ }^{N-1} \xi_{p_{n}}\right\}$. The properties of ${ }^{N-1} \xi_{p_{k}}$ can change on-line based on a given strategy. The stochastic equation (8) for the AOB-1 or Eq. 10 for the AOB-N is used to describe $p_{k}$. If ${ }^{N-1} \xi_{p_{k}}=0$, Eq. 10 is a deterministic model for any disturbance $p_{k}$ that has its $N^{t h}$-derivative equal to zero. In this way, the stochastic information present in ${ }^{N-1} \xi_{p_{k}}$ gives more flexibility to $p_{k}$, since its evolutionary model is not rigid. The estimation of unknown functions using Eqs. 8 and 10 is discussed in [12].

\section{AOB-1 Design}

The AOB-1 algorithm is introduced in this section based on a continuous state space description of the system.

\subsection{System Plant}

Discretizing the system plant (without considering disturbances)

$$
\left\{\begin{array}{l}
\frac{\mathrm{d} x_{r}(t)}{\mathrm{d} t}=A x_{r}(t)+B u(t-\tau) \\
y(t)=C_{r} x_{r}(t)
\end{array}\right.
$$

with sampling time $h$ and dead-time

$$
\tau=(d-1) h+\tau^{\prime}, \text { with } 0<\tau^{\prime} \leq h,
$$

the discrete time system

$$
\left[\begin{array}{c}
x_{k}^{\prime} \\
u_{k-d} \\
\vdots \\
u_{k-2} \\
u_{k-1}
\end{array}\right]=\left[\begin{array}{ccccc}
\Phi_{1} & \Gamma_{1} & \Gamma_{0} & \cdots & 0 \\
0 & 0 & 1 & \cdots & 0 \\
\vdots & \vdots & \vdots & \ddots & \vdots \\
0 & 0 & 0 & \cdots & 1 \\
0 & 0 & 0 & \cdots & 0
\end{array}\right]\left[\begin{array}{c}
x_{k-1}^{\prime} \\
u_{k-d-1} \\
\vdots \\
u_{k-3} \\
u_{k-2}
\end{array}\right]+\left[\begin{array}{c}
0 \\
0 \\
\vdots \\
0 \\
1
\end{array}\right] u_{k-1}
$$

\footnotetext{
3 The mathematical notation along the paper is for single input systems. For multiple input systems, $p_{k}$ in Eq. 8 is a column vector with dimension equal to the number of inputs.

$4 p_{k}$ is a random variable and $\left\{p_{n}\right\}$ is a random process.
} 
and

$$
y_{k}=C_{r} x_{r, k}
$$

is obtained. $\Phi_{1}, \Gamma_{0}$ and $\Gamma_{1}$ are given by Eqs. 15 to 17 , respectively [1].

$$
\begin{aligned}
& \Phi_{1}=e^{A h}=\phi(h), \\
& \Gamma_{0}=\int_{0}^{h-\tau^{\prime}} \phi(\lambda) d \lambda B
\end{aligned}
$$

and

$$
\Gamma_{1}=\phi\left(h-\tau^{\prime}\right) \int_{0}^{\tau^{\prime}} \phi(\lambda) d \lambda B
$$

The state $x_{r, k}$ is

$$
x_{r, k}=\left[\begin{array}{lllll}
x_{k}^{\prime} & u_{k-d} & \cdots & u_{k-2} & u_{k-1}
\end{array}\right]^{T},
$$

in which $x_{k}^{\prime}$ is the system state considering no dead-time. Therefore, the $\tau$ of Eq. 11 increases the system order.

\subsection{AOB-1 Algorithm}

From Figure 2 and knowing Eqs. 1 and 8, the augmented state space representation (open loop) $)^{5}$ is

$$
\left[\begin{array}{c}
x_{r, k} \\
p_{k}
\end{array}\right]=\left[\begin{array}{cc}
\Phi_{r} & \Gamma_{r} \\
0 & 1
\end{array}\right]\left[\begin{array}{c}
x_{r, k-1} \\
p_{k-1}
\end{array}\right]+\left[\begin{array}{c}
\Gamma_{r} \\
0
\end{array}\right] u_{k-1}^{\prime}+\left[\begin{array}{c}
\xi_{x_{r, k}} \\
\xi_{p_{k}}
\end{array}\right],
$$

where

$$
\begin{aligned}
u_{k-1}^{\prime}= & r_{k-1}-\left[\begin{array}{ll}
L_{r} & 1
\end{array}\right]\left[\begin{array}{c}
x_{r, k-1} \\
\hat{p}_{k-1}
\end{array}\right], \\
\Phi_{r}= & {\left[\begin{array}{ccccc}
\Phi_{1} & \Gamma_{1} & \Gamma_{0} & \cdots & 0 \\
0 & 0 & 1 & \cdots & 0 \\
\vdots & \vdots & \vdots & \ddots & \vdots \\
0 & 0 & 0 & \cdots & 1 \\
0 & 0 & 0 & \cdots & 0
\end{array}\right] }
\end{aligned}
$$

and

$$
\Gamma_{r}=\left[\begin{array}{lllll}
0 & 0 & \cdots & 0 & 1
\end{array}\right]^{T} .
$$

$L_{r}$ is obtained by any control technique applied to Eq. 13 to achieve a desired closed loop behavior. The measurement equation is

$$
y_{k}=C\left[\begin{array}{c}
x_{r, k} \\
p_{k}
\end{array}\right]+\eta_{k}
$$

5 In this context, open loop means that the state transition matrix does not consider the influence of state feedback. 
with $^{6}$

$$
C=\left[\begin{array}{ll}
C_{r} & 0
\end{array}\right] .
$$

The desired closed loop system appears when $\hat{p}_{k}=p_{k}$, i.e.,

$$
\left[\begin{array}{c}
x_{r, k} \\
p_{k}
\end{array}\right]=\left[\begin{array}{cc}
\Phi_{r}-\Gamma_{r} L_{r} & 0 \\
0 & 1
\end{array}\right]\left[\begin{array}{c}
x_{r, k-1} \\
p_{k-1}
\end{array}\right]+\left[\begin{array}{c}
\Gamma_{r} \\
0
\end{array}\right] r_{k-1}+\left[\begin{array}{c}
\xi_{x_{r, k}} \\
\xi_{p_{k}}
\end{array}\right] .
$$

The state $x_{r, k}$ in Eq. 25 is accurate if most of the modeling errors are merged to $p_{k}$. Hence, $\xi_{x_{r k}}$ should be small compared to $\xi_{p_{k}}$. The state estimation ${ }^{7}$ must consider not only the influence of the uncertainty $\xi_{x_{r, k}}$, but also the deterministic term due to the reference input, the extended state representation and the desired closed loop response. It is given by ${ }^{8}$

$$
\begin{aligned}
{\left[\begin{array}{c}
\hat{x}_{r, k} \\
\hat{p}_{k}
\end{array}\right]=} & {\left[\begin{array}{cc}
\Phi_{r, n}-\Gamma_{r, n} L_{r} & 0 \\
0 & 1
\end{array}\right]\left[\begin{array}{c}
\hat{x}_{r, k-1} \\
\hat{p}_{k-1}
\end{array}\right]+\left[\begin{array}{c}
\Gamma_{r, n} \\
0
\end{array}\right] r_{k-1}+} \\
& +K_{k}\left\{y_{k}-C\left(\left[\begin{array}{cc}
\Phi_{r, n}-\Gamma_{r, n} L_{r} & 0 \\
0 & 1
\end{array}\right]\left[\begin{array}{c}
\hat{x}_{r, k-1} \\
\hat{p}_{k-1}
\end{array}\right]+\left[\begin{array}{c}
\Gamma_{r, n} \\
0
\end{array}\right] r_{k-1}\right)\right\} .
\end{aligned}
$$

$K_{k}$ is

$$
K_{k}=P_{1 k} C^{T}\left[C P_{1 k} C^{T}+R_{k}\right]^{-1},
$$

and

$$
P_{1 k}=\Phi_{n} P_{k-1} \Phi_{n}^{T}+Q_{k}
$$

with

$$
P_{k}=P_{1 k}-K_{k} C P_{1 k} .
$$

$\Phi_{n}$ is the augmented open loop matrix used in the design,

$$
\Phi_{n}=\left[\begin{array}{cc}
\Phi_{r, n} & \Gamma_{r, n} \\
0 & 1
\end{array}\right] \text {. }
$$

$Q_{k}$ is the system noise matrix, ${ }^{9}$

$$
Q_{k}=\left[\begin{array}{cc}
Q_{x_{r, k}} & 0 \\
0 & Q_{p_{k}}
\end{array}\right] .
$$

$R_{k}$ is the measurement noise matrix, $R_{k}=E\left\{\eta_{k} \eta_{k}^{T}\right\} . P_{k}$ is the mean square error matrix. It should be pointed out that $P_{1 k}$ given by Eq. 28 uses $\Phi_{n}$. Defining $x_{k}, L$, $\Gamma$, and $\xi_{x_{k}}$ as

$$
x_{k}=\left[\begin{array}{ll}
x_{r, k} & p_{k}
\end{array}\right]^{T}, L=\left[\begin{array}{ll}
L_{r} & 1
\end{array}\right], \Gamma=\left[\begin{array}{ll}
\Gamma_{r} & 0
\end{array}\right]^{T}
$$

\footnotetext{
$\overline{6}$ The form of $C$ is maintained for the AOB-N, since the augmented states that describe $p_{k}$ are not measured.

7 The classical Kalman filter (CKF) algorithm can be seen in [3].

$8 \Phi_{r, n}$ and $\Gamma_{r, n}$ represent the nominal values of $\Phi_{r}$ and $\Gamma_{r}$, respectively.

9 Further analysis of the $Q_{k}$ matrix is given in Section 4.2.
} 
and

$$
\begin{aligned}
\xi_{x_{k}} & =\left[\xi_{x_{r, k}} \xi_{p_{k}}\right]^{T}, \\
P_{1 k} & =E\left\{\left(x_{k}-\hat{x}_{k}^{-}\right)\left(x_{k}-\hat{x}_{k}^{-}\right)^{T}\right\},
\end{aligned}
$$

where $^{10}$

$$
\hat{x}_{k}^{-}=\Phi_{n} \hat{x}_{k-1}+\Gamma_{n}\left(r_{k-1}-L \hat{x}_{k-1}\right)
$$

and

$$
x_{k}=\Phi x_{k-1}+\Gamma\left(r_{k-1}-L \hat{x}_{k-1}\right)+\xi_{x_{k}} .
$$

The term $\left(r_{k-1}-L \hat{x}_{k-1}\right)$ appears in Eqs. 35 and 36 . Hence, its influence is canceled in Eq. 34. Straightforward analysis of Eqs. 34 to 36 gives Eq. 28.

\section{AOB-N Design}

The AOB-N is discussed in this section enabling stronger nonlinearities to be compensated by $\hat{p}_{k}$. Section 4.1 presents the AOB-N algorithm and Section 4.2 discusses the stochastic structure of AOB matrices.

\subsection{AOB-N Algorithm}

The AOB-1 algorithm has to be slightly changed for the AOB-N. Only the equation of the active state changes, entailing minor modifications in the overall AOB design.

Equation 10 has the following state space representation:

$$
\begin{aligned}
{\left[\begin{array}{c}
p_{k-(N-1)} \\
p_{k-(N-2)} \\
\vdots \\
p_{k-1} \\
p_{k}
\end{array}\right]=} & {\left[\begin{array}{ccccc}
0 & 1 & 0 & \cdots & 0 \\
0 & 0 & 1 & \cdots & 0 \\
\vdots & \vdots & \vdots & \ddots & \vdots \\
0 & 0 & 0 & \cdots & 1 \\
a_{N} & a_{N-1} & a_{N-2} & \cdots & a_{1}
\end{array}\right]\left[\begin{array}{c}
p_{k-N} \\
p_{k-(N-1)} \\
\vdots \\
p_{k-2} \\
p_{k-1}
\end{array}\right]+\left[\begin{array}{c}
0 \\
0 \\
\vdots \\
0 \\
N-1 \xi_{p_{k}}
\end{array}\right], } \\
& \text { with } a_{i}=(-1)^{i+1} \frac{N !}{i !(N-i) !}, i=1, \cdots, N .
\end{aligned}
$$

In compact form, Eq. 37 is represented by

$$
{ }^{N} p_{k}=\Phi_{2,2}{ }^{N} p_{k-1}+\xi^{N} p_{k} .
$$

Equation 19 is now re-written as

$$
\left[\begin{array}{c}
x_{r, k} \\
{ }^{N} p_{k}
\end{array}\right]=\left[\begin{array}{cc}
\Phi_{r} & \Phi_{1,2} \\
0 & \Phi_{2,2}
\end{array}\right]\left[\begin{array}{c}
x_{r, k-1} \\
{ }^{N} p_{k-1}
\end{array}\right]+\left[\begin{array}{c}
\Gamma_{r} \\
0
\end{array}\right] u_{k-1}^{\prime}+\left[\begin{array}{c}
\xi_{x_{r, k}} \\
\xi_{N} p_{k}
\end{array}\right],
$$

where

$$
{ }^{N} p_{k}=\left[\begin{array}{lllll}
p_{k-(N-1)} & p_{k-(N-2)} & \cdots & p_{k-1} & p_{k}
\end{array}\right]^{T} .
$$

$10 P_{1 k}$ represents the a-priori error covariance matrix only due to the random source $\xi_{x_{k}}$. Therefore, to compute $P_{1 k}$, the real matrices are equal to the nominal ones $\left(\Phi=\Phi_{n}\right.$ and $\left.\Gamma=\Gamma_{n}\right)$. 
$\Phi_{2,2}$ is the state transition matrix of Eq. 37,

$$
\Phi_{1,2}=\left[\begin{array}{ccc}
0 & \cdots & 0 \\
\vdots & \ddots & \vdots \\
0 & \cdots & 1
\end{array}\right]
$$

and

$$
\xi^{N} p_{k}=\left[\begin{array}{lllll}
0 & 0 & \cdots & 0^{N-1} \xi_{p_{k}}
\end{array}\right]^{T} .
$$

The desired closed loop of Eq. 25 is changed to

$$
\left[\begin{array}{c}
x_{r, k} \\
{ }^{N} p_{k}
\end{array}\right]=\left[\begin{array}{cc}
\Phi_{L} & 0 \\
0 & \Phi_{2,2}
\end{array}\right]\left[\begin{array}{c}
x_{r, k-1} \\
N p_{k-1}
\end{array}\right]+\left[\begin{array}{c}
\Gamma_{r} \\
0
\end{array}\right] r_{k-1}+\left[\begin{array}{c}
\xi_{x_{r, k}} \\
\xi_{N} p_{k}
\end{array}\right],
$$

with

$$
\Phi_{L}=\left[\begin{array}{ccccc}
\Phi_{1} & \Gamma_{1} & \Gamma_{0} & \cdots & 0 \\
0 & 0 & 1 & \cdots & 0 \\
\vdots & \vdots & \vdots & \ddots & \vdots \\
0 & 0 & 0 & \cdots & 1 \\
-L_{1} & -L_{2} & -L_{3} & \cdots & -L_{M}
\end{array}\right]
$$

The $L_{r}$ components $\left(L_{1}, \cdots, L_{M}\right)$ can be obtained by Ackermann's formula, i.e.,

$$
L_{r}=\left[\begin{array}{llll}
0 & \cdots & 0 & 1
\end{array}\right] W_{c}^{-1} P\left(\Phi_{r}\right) .
$$

$W_{c}$ is the reachability matrix

$$
W_{c}=\left[\Gamma_{r} \Phi_{r} \Gamma_{r} \cdots \Phi_{r}^{M-1} \Gamma_{r}\right]
$$

and $P\left(\Phi_{r}\right)$ is the desired characteristic polynomial. The state estimation is ${ }^{11}$

$$
\begin{aligned}
& {\left[\begin{array}{c}
\hat{x}_{r, k} \\
{ }^{N} \hat{p}_{k}
\end{array}\right]=\left[\begin{array}{cc}
\Phi_{L, n} & 0 \\
0 & \Phi_{2,2}
\end{array}\right]\left[\begin{array}{c}
\hat{x}_{r, k-1} \\
{ }^{N} \hat{p}_{k-1}
\end{array}\right]+\left[\begin{array}{c}
\Gamma_{r, n} \\
0
\end{array}\right] r_{k-1}} \\
& +K_{k}\left\{y_{k}-C\left(\left[\begin{array}{cc}
\Phi_{L, n} & 0 \\
0 & \Phi_{2,2}
\end{array}\right]\left[\begin{array}{c}
\hat{x}_{r, k-1} \\
N \hat{p}_{k-1}
\end{array}\right]+\left[\begin{array}{c}
\Gamma_{r, n} \\
0
\end{array}\right] r_{k-1}\right)\right\} .
\end{aligned}
$$

$K_{k}$ is given by Eqs. 27 to 29 , with

$$
\Phi_{n}=\left[\begin{array}{cc}
\Phi_{r, n} & \Phi_{1,2} \\
0 & \Phi_{2,2}
\end{array}\right]
$$

The state feedback gain $L$ is

$$
L=\left[\begin{array}{ll}
L_{r} & L_{N} p_{k}
\end{array}\right]
$$

with

$$
L^{N} p_{k}=\left[\begin{array}{llll}
0 & \cdots & 0 & 1
\end{array}\right]
$$

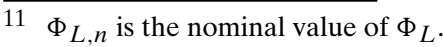




\subsection{AOB-N Matrices}

$R_{k}$ is function of sensor characteristics. The form of $Q_{k}$ is

$$
Q_{k}=\left[\begin{array}{cc}
Q_{x_{r, k}} & 0 \\
0 & Q^{N} p_{k}
\end{array}\right] .
$$

$Q_{x_{r, k}}$ is a diagonal matrix. The uncertainty associated with $x_{r, k}$ is low since all system disturbances should be compensated with ${ }^{N} p_{k}$. Hence, $Q_{x_{r, k}}$ should have low values compared to $Q_{{ } p_{k}}$, which is defined as

$$
Q^{{ } p_{k}}=E\left\{\xi_{N} p_{k} \cdot \xi_{N}^{T} p_{k}\right\}
$$

From Eq. 42,

$$
Q^{N p_{k}}=\left[\begin{array}{ccc}
0 & \cdots & 0 \\
\vdots & \ddots & \vdots \\
0 & \cdots & \sigma_{N-1}^{2} \xi_{p_{k}}
\end{array}\right] .
$$

$\sigma_{N-1 \xi_{p_{k}}}^{2}$ represents the variance of the $N^{\text {th }}$ derivative of $p_{k}$ and is related with $\sigma_{\xi_{p_{k}}}^{2}$. Hence, the design can be done for $\sigma_{\xi_{p_{k}}}^{2}$.

Theorem 1 If the variables $\xi_{p_{k}}$ are independent with zero mean, the relation between $\sigma_{N_{\xi_{k}}}^{2}$ and $\sigma_{\xi_{p_{k}}}^{2}$ is

$$
\sigma_{N_{\xi_{k}}}^{2}=\sigma_{\xi_{p_{k}}}^{2} \sum_{j=0}^{N}\left(\frac{N !}{j !(N-j) !}\right)^{2} .
$$

Proof See [12]

To improve relative stability (discussed in Section 6) for higher order AOBs, Eq. 54 may change to

$$
\sigma_{N \xi_{p_{k}}}^{2}=\sigma_{\xi_{p_{k}}}^{2} \sum_{j=0}^{N}\left(\frac{N !}{j !(N-j) !}\right)^{2} \cdot c^{N},
$$

with

$$
0<c<1 \text {. }
$$

From Eqs. 55 to 56, improving stability is made at the expense of decreasing the "activeness" of active state $p_{k}$.

\section{AOB Estimation Strategies}

Multiplying the matrices $Q_{k}, R_{k}$ and $P_{-1}$ by the same positive factor entails the same Kalman gain [11]. Therefore, the estimation strategy only depends on the relations between the $Q_{k}$ values, the $R_{k}$ values, and both $Q_{k}$ and $R_{k}$ values. In the AOB, the relations between the $Q_{k}$ values are straightforward. The estimation strategy Springer 
is thus a function of $\sigma_{N-1}^{2} \xi_{p_{k}}$ and $R_{k}$. If model accuracy is very good compared to measure accuracy, a model-based approach (MBA) is followed. The estimation is mainly based on model information giving little importance to measures. The Kalman gain has low values. On the other hand, if the measures are very accurate, a sensorbased approach (SBA) is followed. The Kalman gain has high values. The hybridbased approach (HBA) is the general form of the AOB and establishes a trade-off between SBA and MBA, i.e., it balances the estimates based on sensory and model information.

\section{AOB Stability}

This section analyzes AOB absolute/relative stability with model errors.

\subsection{Absolute Stability with Model Errors}

Theorem 2 If a linear system with equations

$$
x_{k}=\Phi x_{k-1}+\Gamma u_{k-1}+\xi_{x_{k}}
$$

and

$$
y_{k}=C x_{k}+\eta_{k}
$$

is being controlled through an observer, where the real system matrix $\Phi$ is equal to the nominal matrix $\Phi_{n}$ plus the unknown error $\Delta \Phi$, i.e., $\Phi=\Phi_{n}+\Delta \Phi$, and, in the same way, the command matrix $\Gamma=\Gamma_{n}+\Delta \Gamma$, the overall system can be described in state space by

$$
\begin{aligned}
{\left[\begin{array}{c}
x_{k} \\
e_{k}
\end{array}\right]=} & {\left[\begin{array}{c}
\Phi-\Gamma L \\
\left(I-K_{k} C\right)(\Delta \Phi-\Delta \Gamma L)\left(I-K_{k} C\right)\left(\Phi_{n}+\Delta \Gamma L\right)
\end{array}\right]\left[\begin{array}{c}
x_{k-1} \\
e_{k-1}
\end{array}\right] } \\
& +\left[\begin{array}{c}
\Gamma \\
\left(I-K_{k} C\right) \Delta \Gamma
\end{array}\right] r_{k-1}+\left[\begin{array}{c}
I \\
I-K_{k} C
\end{array}\right] \xi_{x_{k}}+\left[\begin{array}{c}
0 \\
-K_{k}
\end{array}\right] \eta_{k},
\end{aligned}
$$

with the estimation error

$$
e_{k}=x_{k}-\hat{x}_{k}
$$

$K_{k}, L, I$ and $C, \xi_{x_{k}}$ and $\eta_{k}$ are, respectively, the observer gain, state feedback gain, identity matrix, measurement matrix, system noise and measurement noise. ${ }^{12}$

Proof The command input is

$$
u_{k-1}=r_{k-1}-L \hat{x}_{k-1} .
$$

12 In this analysis, it is considered that the error in $y_{k}$ is only due to $\eta_{k}$, which is the common situation in practice. Hence, no bias terms are considered and $\Delta C=0$. 
Using Eqs. 60 and 61, Eq. 57 can be written as

$$
x_{k}=(\Phi-\Gamma L) x_{k-1}+\Gamma L e_{k-1}+\Gamma r_{k-1}+\xi_{x_{k}} .
$$

A generic observer of Eq. 62 is

$$
\hat{x}_{k}=\left(\Phi_{n}-\Gamma_{n} L\right) \hat{x}_{k-1}+\Gamma_{n} r_{k-1}+K_{k}\left\{y_{k}-C\left[\left(\Phi_{n}-\Gamma_{n} L\right) \hat{x}_{k-1}+\Gamma_{n} r_{k-1}\right]\right\} .
$$

Manipulating Eqs. 62 and 63, Eq. 59 is obtained.

In the AOB-N context,

$$
x_{k}=\left[x_{r, k}{ }^{N} p_{k}\right]^{T} .
$$

From Eq. 59, it is possible to know if the system is stable when model errors exist in the design. Trial and error procedures can be applied to obtain the maximum admissible mismatch that the whole system is able to cope, without loosing stability or without affecting significantly the desired closed loop dynamics. Only the $\Delta$ matrices have to be calculated for some parameter mismatches. Then, stability is assured if the eigenvalues of the system matrix of Eq. 59 lie within the unitary circle.

\subsection{Relative Stability with Model Errors}

Theorem 3 If the linear system of Eqs. 57 and 58 is being controlled through an observer, the loop transfer function (LTF) is

$$
H_{\mathrm{LTF}}(z)=\left[L \begin{array}{ll}
L & 0
\end{array}\right]\left[I-\phi z^{-1}\right]^{-1} \gamma z^{-1},
$$

in which $\phi$ and $\gamma$ are the state transition and command matrices of Eq. 66, respectively, $I$ is the identity matrix and $L$ is the state feedback gain.

$$
\begin{aligned}
{\left[\begin{array}{c}
\hat{x}_{k} \\
e_{k}
\end{array}\right]=} & {\left[\begin{array}{cc}
\Phi_{n}-\Gamma_{n} L+K_{k} C\left(\Delta \Phi+\Gamma_{n} L\right) & K_{k} C \Phi \\
\left(I-K_{k} C\right)\left(\Delta \Phi+\Gamma_{n} L\right) & \left(I-K_{k} C\right) \Phi
\end{array}\right]\left[\begin{array}{c}
\hat{x}_{k-1} \\
e_{k-1}
\end{array}\right] } \\
& +\left[\begin{array}{c}
K_{k} C \Gamma \\
\left(I-K_{k} C\right) \Gamma
\end{array}\right] u_{k-1} .
\end{aligned}
$$

Proof A schematic representation of the LTF is depicted in Figure 3. From Figure 3a,

$$
\hat{x}_{k}=\Phi_{n} \hat{x}_{k-1}+\Gamma_{n} u_{k-1}^{\prime}+K_{k}\left[y_{k}-C\left(\Phi_{n} \hat{x}_{k-1}+\Gamma_{n} u_{k-1}^{\prime}\right)\right],
$$

which is equivalent to $\hat{x}_{k}$ in Figure $3 b$,

$$
\hat{x}_{k}=\left(\Phi_{n}-\Gamma_{n} L\right) \hat{x}_{k-1}+K_{k}\left[y_{k}-C\left(\Phi_{n}-\Gamma_{n} L\right) \hat{x}_{k-1}\right],
$$

with $r_{k}=0$. Hence, both systems have the same LTF form. The matrices $\Phi_{n}$ and $\Gamma_{n}$ are different if the AOB is used (extended matrices). Manipulating Eqs. 57, 60 and 67, Eq. 66 is obtained. The output of the LTF is

$$
Y_{k}=\left[\begin{array}{ll}
L & 0
\end{array}\right]\left[\begin{array}{l}
\hat{x}_{k} \\
e_{k}
\end{array}\right] .
$$

The transfer function $H_{\mathrm{LTF}}(z)$ is then given by Eq. 65 . 

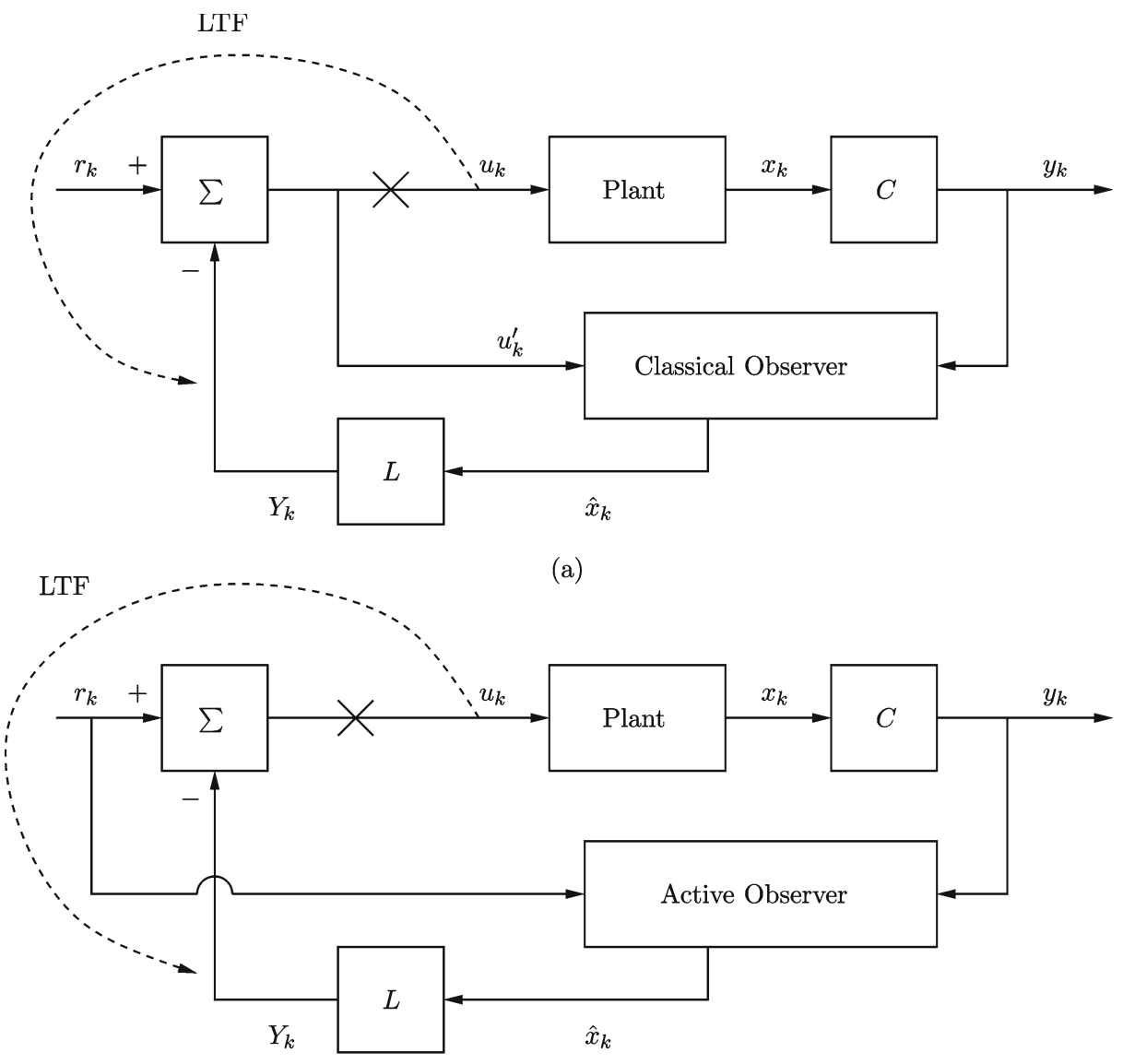

(b)

Figure 3 LTF for systems with classical observer (a) and AOB (b). The reference $r_{k}$ does not enter in the LTF computation $\left(r_{k}=0\right)$.

Therefore, for a given mismatch it is possible to compute relative stability. ${ }^{13}$ As the AOB order increases, relative stability decreases, since the LTF has more poles at $z \sim=\sim 1$ due to the integral action of Eq. 10. An interesting aspect of the AOB architecture is that phase and gain margins can be shaped by the statistics of $Q_{k}$ and $R_{k}$, without changing the control structure. ${ }^{14}$

13 A robotic example can be seen in [13], where relative stability is computed based on the stiffness mismatch.

14 In general, any observer-based system deteriorates relative stability regardless of the position of the observer poles [21]. An example in [14], shows that making the observer poles faster degrades even more stability margins. 


\section{Example 1}

This example shows the design of AOB based controllers for robotic manipulators in contact tasks (SISO system for each degree of freedom). The control performance is analyzed for the AOB-1, AOB-2 and AOB-3 in the presence of an external and unknown nonlinear disturbance.

\subsection{Cartesian Control of Robotic Manipulators}

Given a set of generalized coordinates $q$ (usually, joint angles for revolute joints) describing the robot's pose, the well-known robot dynamics is given by

$$
M(q) \ddot{q}+v(q, \dot{q})+g(q)=\tau .
$$

$M(q)$ is the mass matrix, $v(q, \dot{q})$ is the vector of Coriolis and centripetal forces, $g(q)$ is the gravity term and $\tau$ is the generalized torque acting on $q$. Defining the Jacobian $J(q)$ as

$$
\dot{X}=J(q) \dot{q}
$$

with $X$ the Cartesian position,

$$
\ddot{q}=J^{-1}(\ddot{X}-\dot{J} \dot{q}) .
$$

Applying Eq. 72 in Eq. 70 and knowing the relation between the joint torque $\tau$ and the Cartesian force $F$ at the end-effector, $\tau=J^{T} F$, Eq. 70 can be written in Cartesian coordinates as

$$
\Lambda \ddot{X}+V_{x}(q, \dot{q})+g_{x}(q)=F,
$$

with

$$
\begin{aligned}
\Lambda & =J^{-T} M J^{-1}, \\
V_{x} & =J^{-T} v(q, \dot{q})-\Lambda \dot{J} \dot{q}
\end{aligned}
$$

and

$$
g_{x}=J^{-T} g(q) .
$$

An external force $F_{e}$ appears always at the end-effector whenever the robot is in contact. Hence, Eq. 73 can be written as

$$
\Lambda \ddot{X}+V_{x}(q, \dot{q})+g_{x}(q)=F_{c}-F_{e}-F_{f},
$$

where $F_{c}$ and $F_{f}$ are, respectively, the forces due to the commanded torque and friction. Applying the feedback linearization control law ${ }^{15}$

$$
F_{c}=\hat{F}_{e}+\hat{F}_{f}+\hat{V}_{x}(q, \dot{q})+\hat{g}_{x}(q)+\hat{\Lambda} f^{\star}
$$

to Eq. 77 , the desired decoupled control system

$$
\ddot{X}=f^{\star}
$$

15 The symbol` means estimate.

를 Springer 


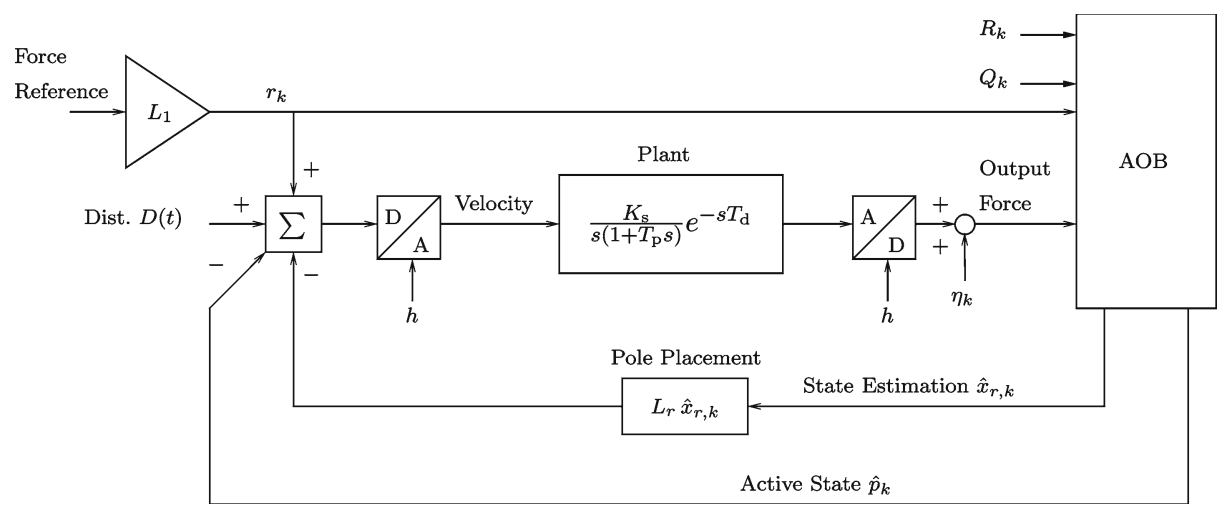

Figure 4 Force control with AOB.

is obtained. Inserting the velocity feedback, the system deadtime $T_{d}$ and the system stiffness $K_{\mathrm{s}}$ in Eq. 79, each Cartesian degree of freedom can be described by

$$
G_{\mathrm{v}}(s)=\frac{K_{\mathrm{s}}}{s\left(1+T_{\mathrm{p}} s\right)} e^{-s T_{\mathrm{d}}}
$$

where $T_{\mathrm{p}}$ is the position time constant. Figure 4 represents the force control of Eq. 80 with AOB.

\subsection{Simulation Results}

Figure 5 illustrates simulation results considering the influence of a nonlinear exogenous disturbance $D(t)$ added to the system input. The disturbance is

$$
D(t)=u^{3}+u^{2}-u
$$

with $^{16}$

$$
u=2 \operatorname{Chirp}(0.01[H z], 0.1[H z], 20[s])-0.5 \operatorname{Step}(t-8) .
$$

In this example there are no explicit modeling errors, i.e., the nominal parameters that characterize the system are equal to the real ones. Hence, the dominant disturbance is given by Eq. 81 . The sampling time $h=8[\mathrm{~ms}]$, the deadtime $T_{d}=5 h$, the system stiffness $K_{\mathrm{s}}=3[\mathrm{~N} / \mathrm{mm}]$ and $T_{\mathrm{p}}=32[\mathrm{~ms}]$. The feedback gain $L_{r}\left(L_{1}, \cdots, L_{M}\right.$ with $M=7)$ for a critically damped response $(\zeta=1)$ with time constant $\tau_{c}=10 T_{\mathrm{p}}$ and five extra poles at $z=0$ is

$$
L_{r}=[0.1148-0.0717-0.0615-0.0797-0.1031-0.1332-0.1718] .
$$

16 The Chirp function is a sine wave with increasing frequency. 


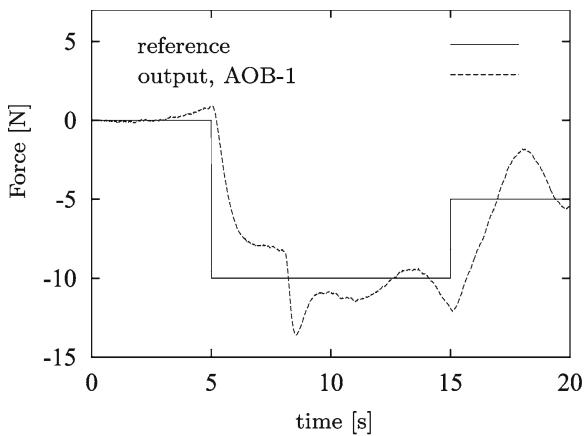

(a)

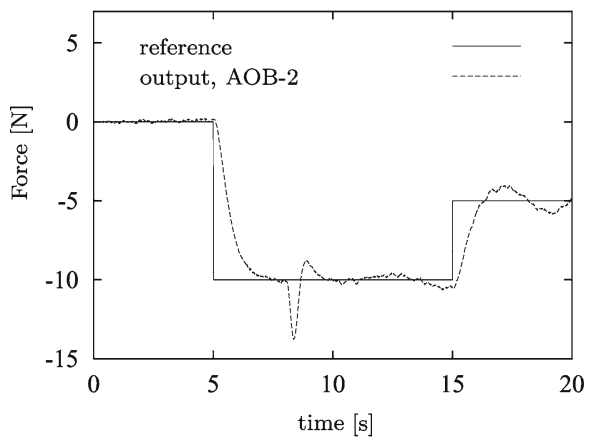

(c)

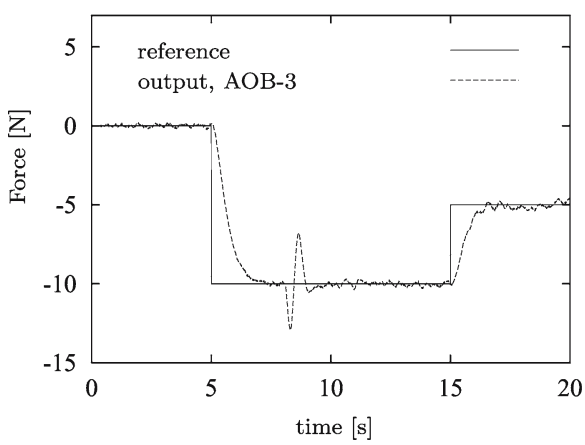

(e)

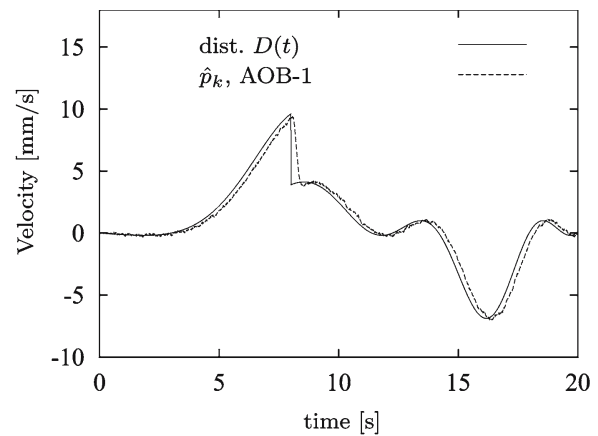

(b)

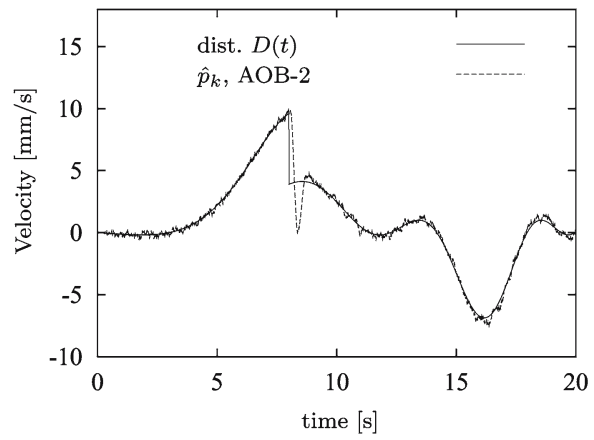

(d)

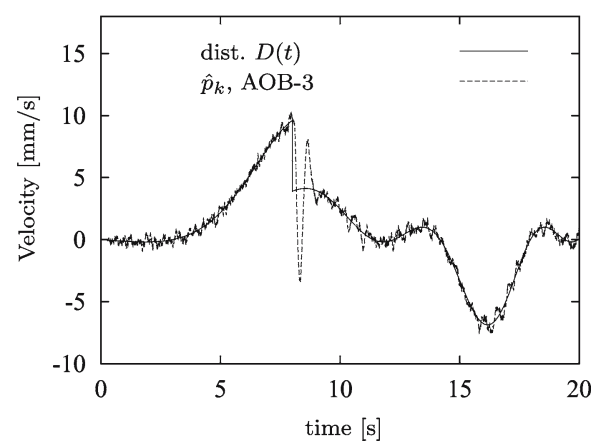

(f)

Figure 5 Simulation results for different AOBs. a Force response with AOB-1. b Disturbance estimation with AOB-1. c Force response with AOB-2. d Disturbance estimation with AOB-2. e Force response with AOB-3. f Disturbance estimation with AOB-3.

The measurement noise $\eta_{k}$ has a power of $R_{k}=2.1 \times 10^{-2}\left[\mathrm{~N}^{2}\right] \cdot Q_{x_{r, k}}$ is a diagonal matrix given by $Q_{x_{r, k}}(i, i)=10^{-12}$, with $i=1, \cdots, M$. The variance of $\xi_{p_{k}}$ is $\sigma_{\xi_{p_{k}}}^{2} \sim=$ $\sim 10^{-3}$ and $c=1 / 500$. Using Eq. 55,

$$
\sigma_{N_{\xi_{p_{k}}}}^{2}=10^{-3} \cdot \sum_{j=0}^{N}\left(\frac{N !}{j !(N-j) !}\right)^{2} \cdot(1 / 500)^{N} .
$$


Table I Steady-state Kalman gain for AOB-1, AOB-2 and AOB-3

The bold values affect ${ }^{N} \hat{p}_{k}$. The other values enter in the estimate of $\hat{x}_{r, k}$, where

$x_{k}^{\prime}=\left[\begin{array}{ll}x_{1 k}^{\prime} & x_{2_{k}}^{\prime}\end{array}\right]^{T} \cdot x_{1 k}^{\prime}$ and $x_{2_{k}}^{\prime}$ represent the output force and output force derivative, respectively.

\begin{tabular}{cccc}
\hline & AOB-1 & AOB- & AOB-3 \\
\hline$K_{k}$ & 0.0959 & 0.1285 & 0.1691 \\
0.6043 & 1.1033 & 1.9533 \\
0.2073 & 0.4256 & 0.8134 \\
0.2073 & 0.4385 & 0.8536 \\
0.2073 & 0.4514 & 0.8949 \\
0.2073 & 0.4643 & 0.9371 \\
0.2073 & 0.4771 & 0.9803 \\
& $\mathbf{0 . 2 0 7 3}$ & $\mathbf{0 . 4 7 7 1}$ & $\mathbf{0 . 9 3 7 1}$ \\
& & $\mathbf{0 . 4 9 0 0}$ & $\mathbf{0 . 9 8 0 3}$ \\
& & & $\mathbf{1 . 0 2 4 5}$ \\
\hline
\end{tabular}

$Q^{N} p_{k}$ is given by Eq. 53. The initial conditions are $\hat{x}_{r, k}=0,{ }^{N} \hat{p}_{k}=0$ and $P_{-1}=Q_{0}$. It can be inferred from Table I that the Kalman gain increases with the AOB order. Hence, the state estimates react fast to measures being more sensitive to noise. It is notorious the improvement of the force response as the AOB order increases. In this example, the disturbance $D(t)$ is high with respect to the system input $L_{1} r_{k}$.

\section{Example 2}

This example shows the design of AOB based controllers for SBW vehicles (MIMO system) with underactuated structure. Model-matching is analyzed for the AOB-1, as well as the effect of a road disturbance.

\subsection{SBW Systems}

Teleoperated robotic vehicles can benefit from SBW research. Guiding vehicles with good haptic feeling improves driver's skills, enabling the exploration of unstructured roads. Vehicle steering technology is substituting mechanical and hydraulic subsystems by electrical equivalents to boost performance. SBW introduces complex mechatronical steering technology consisting of processing units, sensors and actuators. The conventional mechanical interface (steering column) between driver and vehicle is replaced by two electrical actuators which are algorithmically coupled by the SBW controller to provide a desired steering behavior. SBW technology has some essential advantages, such as, simplified construction and higher design flexibility. However, the independent torque assistance and active steering functionalities of some steering technologies motivate a part of automotive engineers to reject SBW technology. The main decision factors, such as safety, comfort and costs are still unclear for SBW systems. Therefore, it is difficult to predict when and if ever SBW vehicles will replace vehicles with steering column. Nevertheless, teleguided vehicles are excellent candidates for SBW (or steer-by-wireless) products, enabling the operator to have steering telepresence. SBW systems are basically master-slave systems with small time delay. The driver corresponds to the operator, the vehicle to the environment, the force-feedback actuator to the master and the front-wheel actuator to the slave. From the control point of view, SBW includes a force feedback inner-loop on the master side and a position or force inner-loop on the slave side. 


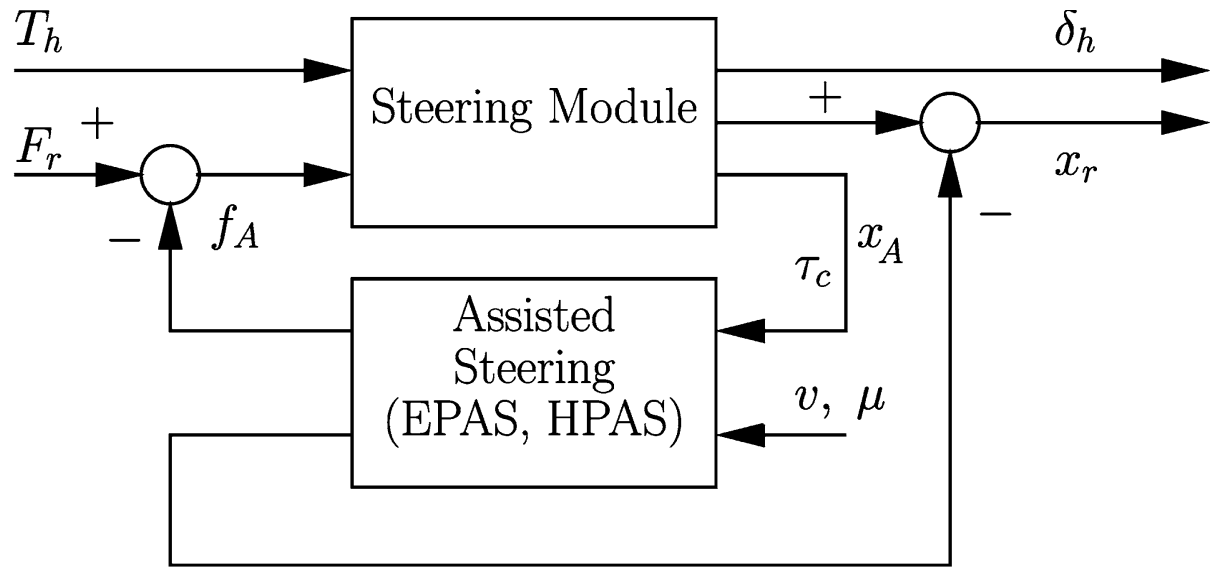

Figure 6 Target system. $F_{r}$ is the road feedback, including unknown disturbances. $T_{h}, f_{A}, F_{r}, v, \mu$, $\tau_{C}, x_{r}, \delta_{h}$ and $x_{A}$ are, respectively, the torque on the steering wheel, assisted steering torque, steering rack force, vehicle speed, tire-road adhesion coefficient, torsional torque on steering column, steering rack position, steering wheel angle, and assisted steering angle.

The goal is to couple these sub-systems by a suitable outer-loop controller to provide a desired steering feeling associated to the vehicle response.

\subsection{Target Dynamics}

A SBW system should reproduce, at least in a first stage, the same dynamics of a steering column car. Some steering systems comprehend algorithms for independent force and position assistance. Usually, the assisted torque steering filter is a nonlinear function of the vehicle speed. The steering angle assistance is introduced to improve lateral and vertical vehicle dynamics. The target system which represents the steering of conventional cars is represented in Figure 6. The steering module includes the steering wheel, steering column, steering rack, power steering actuator and torque sensors, that can be assisted by Electrical and/or Hydraulic Power Assisted Steering (EPAS, HPAS). Linearizing the target system, the overall dynamics can be expressed in the s-domain (i.e., Laplace Transform) by

$$
\left[\begin{array}{l}
s \delta_{h} \\
s x_{r}
\end{array}\right]=\left[\begin{array}{ll}
y_{11} & y_{12} \\
y_{21} & y_{22}
\end{array}\right]\left[\begin{array}{l}
T_{h} \\
F_{r}
\end{array}\right],
$$

where $\delta_{h}, x_{r}, T_{h}$ and $F_{h}$ are Laplace variables, and $y_{11}, y_{12}, y_{21}$ and $y_{22}$ are transfer functions. The SBW technology has to match the target steering dynamics by suitable actuation and control.

\subsection{SBW Dynamics}

The SBW setup controlled by the AOB is represented in Figure 7. It includes two actuation inner-loops for torque and force control. The dynamics of the torque feedback loop can be described by

$$
s \delta_{h}=y_{h}\left(T_{h}-\alpha_{h} \tau_{m}\right) .
$$




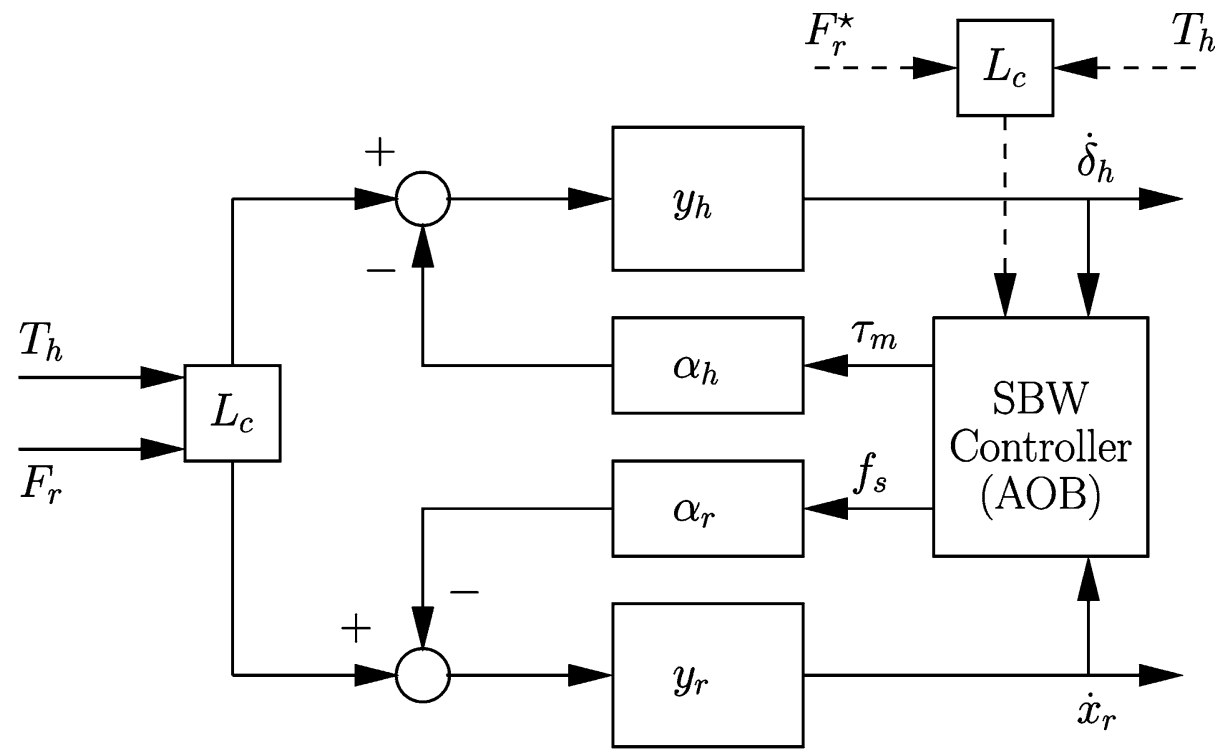

Figure 7 SBW control system with AOB. $F_{r}^{\star}$ is artificially generated (i.e., computed) for a desired vehicle impedance. $F_{r}$ is the real road feedback (steering rack force). $T_{h}$ is measured by a torque sensor on the steering wheel and $L_{c}$ represents the DC gain compensation. $\delta_{h}$ and $x_{r}$ are, respectively, the steering wheel angle and the steering rack position, and $y_{h}, \alpha_{h}, \alpha_{r}$ and $y_{r}$ are transfer functions of the SBW setup.

The actuator, torque sensor and built-in controller are lumped into the transfer functions $y_{h}$ and $\alpha_{h}$. Similarly, the road-wheel actuation loop is described by

$$
s x_{r}=y_{r}\left(F_{r}-\alpha_{r} f_{s}\right) .
$$

Target closed-loop dynamics, desired road feedback $F_{r}^{\star}$ and torque on the steering wheel enter in the AOB design. Disturbance rejection is based on extended states and stochastic design. In the s-domain, the overall open-loop admittance structure is

$$
\left[\begin{array}{l}
s \delta_{h} \\
s x_{r}
\end{array}\right]=\left[\begin{array}{cc}
y_{h} & 0 \\
0 & y_{r}
\end{array}\right]\left(\left[\begin{array}{c}
T_{h} \\
F_{r}
\end{array}\right]-A_{1}\left[\begin{array}{c}
\tau_{m} \\
f_{s}
\end{array}\right]\right),
$$

with

$$
A_{1}=\left[\begin{array}{cc}
\alpha_{h} & 0 \\
0 & \alpha_{r}
\end{array}\right]
$$

\subsection{Model-matching}

This section describes the SBW controller with AOB. The target model representing a conventional vehicle at $v=80[\mathrm{~km} / \mathrm{h}]$ with $\mu=0.8$ is given by [9]

$$
\left[\begin{array}{l}
s \delta_{h} \\
s x_{r}
\end{array}\right]=\left[\begin{array}{llll}
T_{1,1} & 0 & T_{1,3} & 0 \\
T_{2,1} & 0 & T_{2,3} & 0
\end{array}\right]\left[\begin{array}{c}
T_{h} \\
\tau_{m} \\
F_{r} \\
f_{s}
\end{array}\right] .
$$


Since it is not possible to make a direct feedback at the entry points $T_{h}$ and $F_{r}$, four independent inputs (i.e., $T_{h}, \tau_{m}, F_{r}$ and $f_{s}$ ) and two outputs (i.e., $\dot{\delta}_{h}$ and $\dot{x}_{r}$ ) have been used, where

$$
\begin{aligned}
& T_{1,1}=\frac{33.33 s^{2}+44.8 .7 s+93690}{s^{3}+20.71 s^{2}+7655 s+45790}, \\
& T_{1,3}=\frac{1.295 s+894.6}{s^{3}+20.71 s^{2}+7655 s+45790}, \\
& T_{2,1}=\frac{-1.295 s-894.6}{s^{3}+20.71 s^{2}+7655 s+45790}
\end{aligned}
$$

and

$$
T_{2,3}=\frac{-0.001789 s^{2}-0.01296 s-8.543}{s^{3}+20.71 s^{2}+7655 s+45790} .
$$

$\delta_{h}$ and $x_{r}$ are both functions of $T_{h}$ and $F_{r}$. Therefore, the SBW controller has to impose this behavior to the naturally decoupled SBW system (the steering wheel is physically decoupled from the road-wheel actuator). In our setup, the transfer functions $y_{h}, y_{r}, \alpha_{h}$ and $\alpha_{r}$ have been identified (see Figure 7). They are

$$
\begin{aligned}
y_{h} & =\frac{26.67 s^{2}+568 s+218300}{s^{3}+28.1 s^{2}+16430 s+120000}, \\
y_{r} & =\frac{-0.00179}{s+9.395} \\
\alpha_{h} & =\frac{-13510}{s^{2}+21.3 s+8188}
\end{aligned}
$$

and

$$
\alpha_{r}=\frac{1974000}{s+1885}
$$

The model-matching problem consists in designing a state-space controller for the system

$$
\left[\begin{array}{l}
s \delta_{h} \\
s x_{r}
\end{array}\right]=\left[\begin{array}{cccc}
y_{h}-\alpha_{h} y_{h} & 0 & 0 \\
0 & 0 & y_{r} & -\alpha_{r} y_{r}
\end{array}\right]\left[\begin{array}{c}
T_{h} \\
\tau_{m} \\
F_{r} \\
f_{s}
\end{array}\right]
$$

to achieve Eq. 90. Writing Eq. 90 in discrete state-space form with sampling time $T_{s}=1[\mathrm{~ms}]$, the desired closed-loop poles are

$$
\lambda_{1,2}=0.9890 \pm 0.0859 i \text { and } \lambda_{3}=0.9940 .
$$

The discrete state-space of Eq. 99 can be represented by Eq. 19, where $\Phi_{r}$ and $\Gamma_{r}$ have dimensions $7 \times 7$ and $7 \times 4$, respectively. To apply the AOB design described in Section 3, a state feedback matrix $L_{r}$ has to be found, based on Eq. 100 and four additional poles $\left(\lambda_{4} \cdots \lambda_{7}\right)$, due to the dimension of $\Phi_{r}$. There is not a straightforward 
procedure to obtain the additional poles. Simulations have shown that there are many possibilities to achieve good results, such as

$$
\lambda_{4}=0.89, \lambda_{5}=0.89, \lambda_{6}=-0.89 \text { and } \lambda_{7}=0.994 .
$$

The minus sign in one pole is important due to the transient effect of a positive zero in the target system. To compute ${ }^{17} L_{r}, \Gamma_{r}$ has to be changed (only for this computation) to reflect the lack of actuation on the inputs $T_{h}$ and $F_{r}$. The first and third columns of $\Gamma_{r}$ should be set to zero. This guarantees that if there is an $L_{r}$, it will not generate a direct feedback referred to non-actuated inputs. The DC gain of the SBW system is compensated by proper pre-amplification of the reference inputs. For the target system ${ }^{18}$

$$
{ }^{t} \mathrm{DC}=C_{t}\left[I-\left(\Phi_{t}\right)\right]^{-1} \Gamma_{t} .
$$

For the SBW system,

$$
{ }^{\mathrm{SBW}} \mathrm{DC}=C_{\mathrm{SBW}}\left[I-\left(\Phi_{\mathrm{SBW}}-\Gamma_{\mathrm{SBW}} L_{\mathrm{SBW}}\right)\right]^{-1} \Gamma_{\mathrm{SBW}} .
$$

Therefore,

$$
L_{c}=\left({ }^{\mathrm{SBW}} \mathrm{DC}\right)^{-1}{ }^{t} \mathrm{DC} .
$$

In our system, Eq. 104 is

$$
L_{c}=\left[\begin{array}{rr}
0.4785 & -0.0046 \\
67.0076 & -0.6399
\end{array}\right] .
$$

The reference input is then

$$
r_{k}=L_{c}\left[\begin{array}{c}
T_{h} \\
F_{r}
\end{array}\right] .
$$

If input pre-amplification is not possible, Eq. 106 can be obtained from the actuation points by ( $s$-domain)

$$
r_{k}^{\prime}=\left[\begin{array}{c}
T_{h} \\
F_{r}
\end{array}\right]+\left[\begin{array}{cc}
\alpha_{h} & 0 \\
0 & \alpha_{r}
\end{array}\right]^{-1}\left(L_{c}-I\right)\left[\begin{array}{c}
T_{h} \\
F_{r}
\end{array}\right] .
$$

In practice, low-pass filters applied to the inputs are necessary to compute $r_{k}^{\prime}$.

For the SBW setup,

$$
\begin{aligned}
Q_{x_{r, k}} & =10^{-12} I_{7 \times 7}, \\
Q_{p_{k}} & =\left[\begin{array}{cccc}
0 & 0 & 0 & 0 \\
0 & 10^{-1} & 0 & 0 \\
0 & 0 & 0 & 0 \\
0 & 0 & 0 & 10^{3}
\end{array}\right],
\end{aligned}
$$

$R_{k}=4 I_{2 \times 2}$ and $P_{-1}=Q_{0}$. Since there are four inputs, $p_{k}$ has four states,

$$
p_{k}=\left[\begin{array}{llll}
p_{1, k} & p_{2, k} & p_{3, k} & p_{4, k}
\end{array}\right]^{T} \text {. }
$$

\footnotetext{
17 The place command in matlab ${ }^{\mathrm{TM}}$ does the job.

18 The notation ' $t$ ' means target system and $I$ is the identity matrix.
} 
$\hat{p}_{1, k}$ and $\hat{p}_{3, k}$ cannot enter in the system (there is no actuation entry). Hence, the first and third lines of $Q_{p_{k}}$ are zero. This design locks the estimation of $p_{1, k}$ and $p_{3, k}$ $\left(\hat{p}_{1, k}=0\right.$ and $\hat{p}_{3, k}=0$ ). From Eq. 109, the uncertainty of $p_{4, k}$ is higher, enabling $\hat{p}_{4, k}$ to have faster dynamics.

\subsection{Simulation Results}

This section reports simulation results, comparing the performance of the target system vs. the SBW controller with AOB. Applying a torque on the steering wheel (Figure 8a), the steering angle $\delta_{h}$ and the rack position $x_{r}$ are depicted in Figure 8c and d, respectively, for the SBW system (solid lines) and for the conventional vehicle (dashed lines). It can be inferred that model matching was accomplished. Figure $8 \mathrm{~b}$ represents the road feedback. $F_{r}$ can be computed based on the target

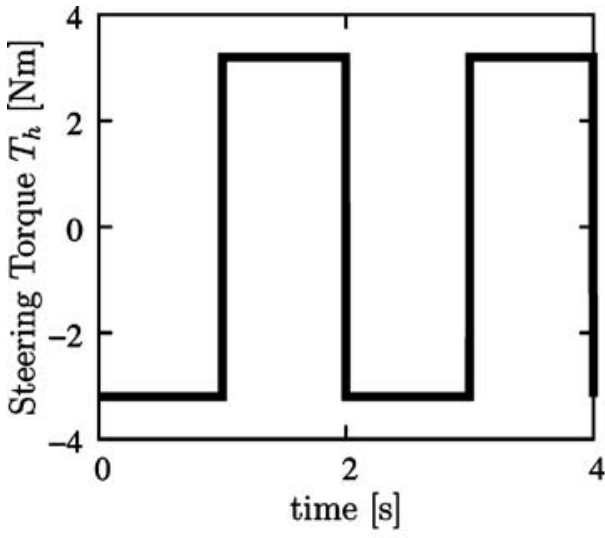

(a)

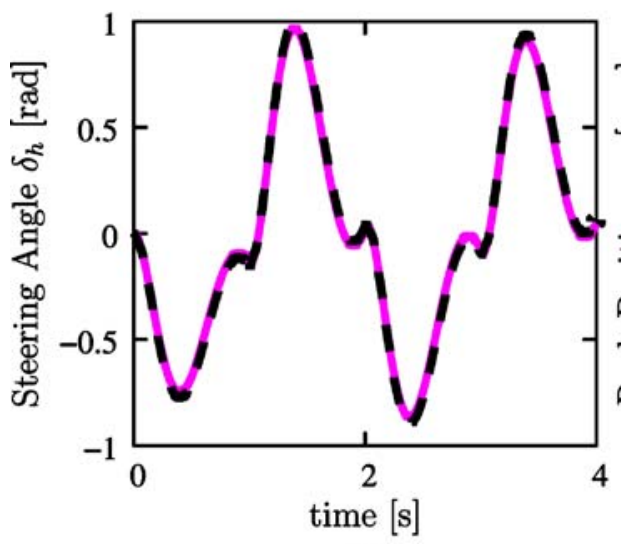

(c)

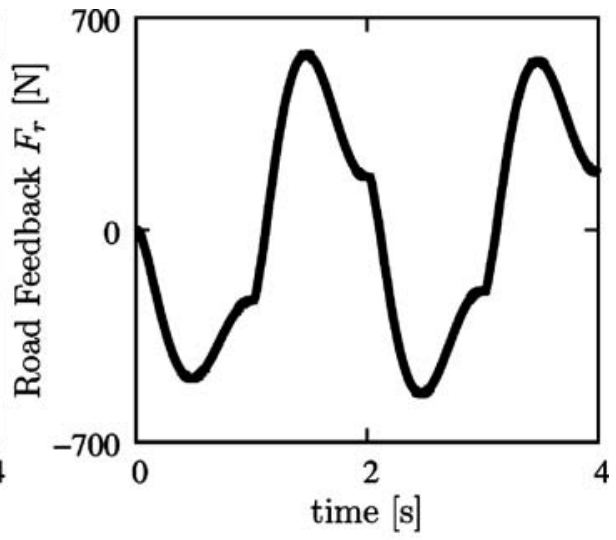

(b)

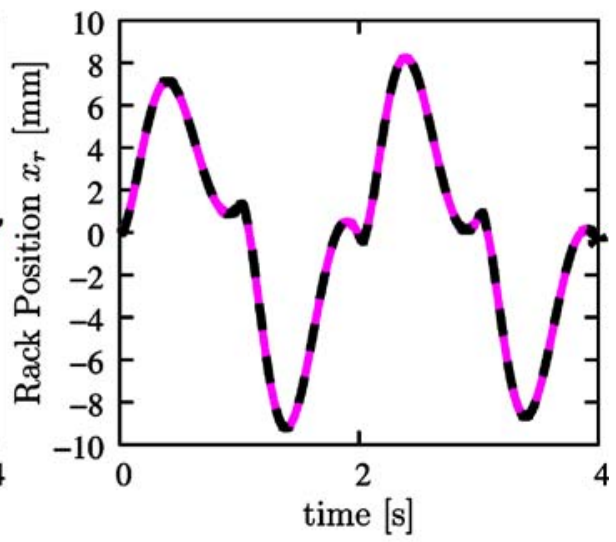

(d)

Figure $8 \mathrm{SBW}$ with AOB vs. conventional car. Model matching data. a Torque input $T_{h}$. b Road feedback $F_{r}$ based on desired vehicle impedance $\left(F_{r}=F_{r}^{\star}\right)$. c $\delta_{h}$ for SBW (solid line) and conventional vehicle (dashed line). d $x_{r}$ for SBW (solid line) and conventional vehicle (dashed line). 


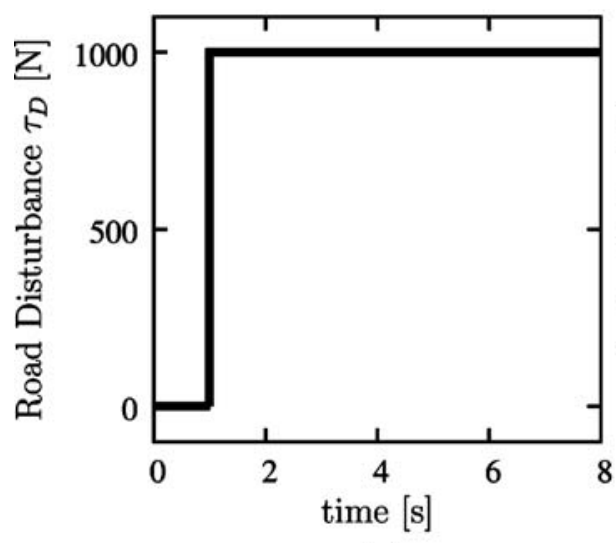

(a)

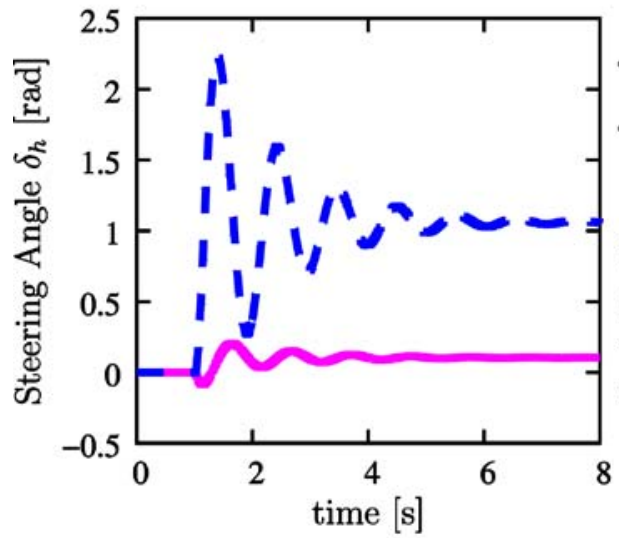

(c)

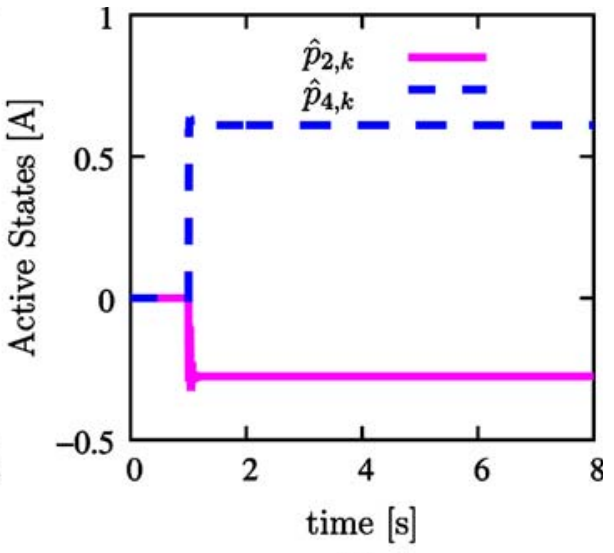

(b)

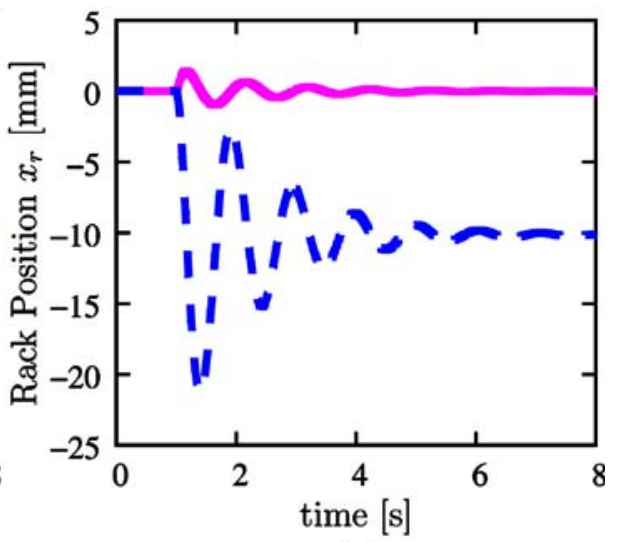

(d)

Figure 9 SBW with AOB vs. conventional car in the presence of road disturbances. a Road disturbance $\tau_{D}=1000[\mathrm{~N}]$ applied at $1[\mathrm{~s}] . \mathbf{b}$ Active state data $\left(\hat{p}_{2, k}\right.$ and $\left.\hat{p}_{4, k}\right)$. $\mathbf{c} \delta_{h}$ for SBW (solid line) and conventional vehicle (dashed line). $\mathbf{d} x_{r}$ for SBW (solid line) and conventional vehicle (dashed line).

vehicle impedance, which is of form [2]

$$
Z_{v}(s)=\frac{a_{2} s^{2}+a_{1} s+a_{0}}{s\left(b_{2} s^{2}+b_{1} s+b_{0}\right)}
$$

The AOB structure may use this information to compensate all other road disturbances. This situation is analyzed in Figure 9. For a step disturbance (see Figures 7 and $9 \mathrm{a}$ )

$$
\tau_{D}=F_{r}-F_{r}^{\star}
$$


applied at 1 [s], the active states $\hat{p}_{2, k}$ and $\hat{p}_{4, k}$ compensate it very fast (Figure 9b). These states converge to the values

$$
\left[\begin{array}{c}
\hat{p}_{2, k} \\
\hat{p}_{4, k}
\end{array}\right] \rightarrow-\left[\begin{array}{cc}
\alpha_{h} & 0 \\
0 & \alpha_{r}
\end{array}\right]_{s=0}^{-1} \cdot L_{c}\left[\begin{array}{c}
0 \\
\tau_{D}
\end{array}\right],
$$

that is,

$$
\left[\begin{array}{l}
\hat{p}_{2, k} \\
\hat{p}_{4, k}
\end{array}\right] \rightarrow\left[\begin{array}{r}
-0.27 \\
0.61
\end{array}\right] .
$$

On the contrary of conventional car, the steering angle has a small deviation and the rack position is not affected by steady-state errors (Figure 9c and d).

\section{Conclusions}

The AOB has been introduced in this paper. The main goal is to achieve modelreference adaptive control using Kalman techniques. The AOB design is based on: (1) A desired closed loop system. (2) An extra equation (active state) to estimate an equivalent disturbance referred to the system input, providing a feedforward compensation action. (3) The stochastic design of the Kalman matrices $Q_{k}$ and $R_{k}$.

The active state is modeled as an auto-regressive process of order $N$ with fixed parameters (evolutions) driven by a Gaussian random source. In the AOB, $Q_{k}$ has a well defined structure. Most of the modeling errors are merged in the active state, therefore, the model for the other states is very accurate (the reference model has low uncertainty). The relation between $Q_{k}$ and $R_{k}$ defines the estimation strategy, making the state estimates more or less sensitive to measures. Increasing the AOB order increases the capability to track higher nonlinear disturbances, but the sensitivity to noise and computational effort increase and the relative stability and robustness decrease. Re-adapting the stochastic strategies for higher order AOBs may improve relative stability margins. The design of AOB based controllers for robotic manipulators in contact (SISO system) and for SBW vehicles (MIMO system) have been discussed. Simulation results have shown the AOB performance in the presence of external disturbances.

\section{References}

1. Aström, K.J., Wittenmark, B.: Computer Controlled Systems: Theory and Design. Prentice Hall, NJ (1997)

2. Bajcinca, N., Cortesão, R., Hauschild, M.: Robust control for steer-by-wire vehicles. Auton. Robots 19 193-214 (2005)

3. Bozic, S.M.: Digital and Kalman Filtering. Edward Arnold, London (1979)

4. Chen, B., Lin, Z., Liu, K.: Robust and perfect tracking of discrete time systems. Automatica 38(2), 293-299 (2002)

5. Chen, X., Komada, S., Fukuda, T.: Design of a nonlinear disturbance observer. IEEE Trans. Ind. Electron. 47(2), 429-435 (2000)

6. Chu, D., Mehrmann, V.: Disturbance decoupling for descriptor systems by state feedback. SIAM J. Control Optim. 38(6), 1830-1858 (2000)

7. Coelho, P., Nunes, U.: Path-following control of mobile robots in presence of uncertainties. IEEE Trans. Robot. 21(2), 252-261 (2005)

8. Commault, C., Dion, J., Hovelaque, V.: A geometric approach for structured systems - application to the disturbance decoupling problem. Automatica 33(3), 403-409 (1997) 
9. Cortesão, R., Bajcinca, N.: Model-matching control for steer-by-wire vehicles with underactuated structure. In: Proc. of the Int. Conf. on Intelligent Robots and Systems (IROS), Japan, pp. 1148-1153 (2004)

10. Cortesão, R., Koeppe, R., Nunes, U., Hirzinger, G.: Force control with a Kalman active observer applied in a robotic skill transfer system. Int. J. Machine Intel. Robot. Control (MIROC). 2(2), 59-68 (2000), Special Issue on Force Control of Advanced Robotic Systems

11. Cortesão, R., Koeppe, R., Nunes, U., Hirzinger, G.: Compliant motion control with stochastic active observers. In: Proc. of the Int. Conf. on Intelligent Robots and Systems (IROS), USA, pp. 1876-1881 (2001)

12. Cortesão, R., Koeppe, R., Nunes, U., Hirzinger, G.: Data fusion for robotic assembly tasks based on human skills. IEEE Trans. Robot. 20(6), 941-952 (2004)

13. Cortesão, R., Park, J., Khatib, O.: Real-time adaptive control for haptic telemanipulation with Kalman active observers. IEEE Trans. Robot. 22(5) 987-999 (October 2006)

14. Doyle, J., Stein, G.: Robustness with observers. IEEE Trans. Automat. Contr. 24(4), 607-611 (1979)

15. Estrada, M., Malabre, M.: Necessary and sufficient conditions for disturbance decoupling with stability using PID control laws. IEEE Trans. Automat. Contr. 44(6), 1311-1315 (1999)

16. Maia, R., Cortesão, R., Nunes, U., Silva, V., Fonseca, F.: Robust low level motion control of a WMR with stochastic active observers. In: Proc. of the Int. Conf. on Advanced Robotics, Portugal, pp. 876-882 (2003)

17. Oda, N.: Distributed robust motion controller for redundant manipulator using disturbance observer. J. Robot. Mechatronics 13(5), 464-471 (2001)

18. Park, J., Cortesão, R., Khatib, O.: Multi-contact compliant motion control for robotic manipulators. In: Proc. of the IEEE Int. Conf. on Robotics and Automation, (ICRA), USA, pp. 4789-4794 (2004)

19. Park, J., Khatib, O.: Multi-link multi-contact force control for manipulators. In: Proc. of the Int. Conf. on Robotics and Automation (ICRA), Spain, pp. 3624-3629 (2005)

20. De Schutter, J.: Improved force control laws for advanced tracking applications. In: Proc. of the Int. Conf. on Robotics and Automation (ICRA), USA, pp. 1497-1502 (1988)

21. Vaccaro, R.: Digital Control: A State-Space Approach. McGraw-Hill, New York (1995) 